\title{
RECHNUNGSLEGUNG
}

\section{Entwarnung für Leistungserbringer}

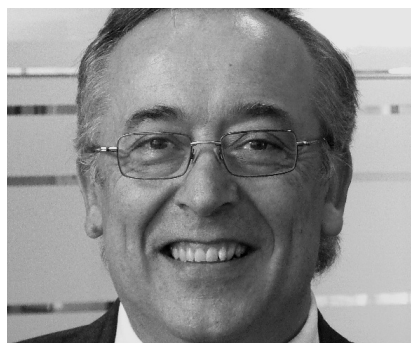

VON FRIEDRICH DICKOPP Friedrich Dickopp ist als Wirtschaftsprüfer und Steuerberater bei der Solidaris Revisions- $\mathrm{GmbH}$, einer mittelgroßen Wirtschaftsprüfungs- und Steuerberatungsgesellschaft tätig, die sich auf die Betreuung von Mandanten des Dritten Sektors konzentriert. Er ist Mitglied im Arbeitskreis »Besteuerung von Non-Profit-Organisationen « des Instituts der Wirtschaftsprüfer in Deutschland e. V. (IDW) und im Arbeitskreis "Gemeinnützigkeitsrecht" der Bundesarbeitsgemeinschaft der Freien Wohlfahrtspflege e. V.

E-Mail f.dickopp@solidaris.de

\begin{abstract}
Die neuen Bestimmungen zur Rechnungslegung Spenden sammelnder Organisationen haben für Unsicherheiten bei einigen - insbesondere kleineren - Trägern sozialer Dienste und Einrichtungen gesorgt, da sie erheblichen Mehraufwand bedeuten würden. Doch sind von den neuen Vorgaben Organisationen gar nicht betroffen, die ihre Arbeit hauptsächlich aus Leistungsentgelten finanzieren.
\end{abstract}

Im vorigen Jahr hat der Hauptfachausschuss des Instituts der Wirtschaftsprüfer in Deutschland e. V. eine Stellungnahme zur Rechnungslegung zu den »Besonderheiten der Rechnungslegung Spenden sammelnder Organisationen « (IDW RS HFA 21) verabschiedet. Darin wird deutlich herausgestellt, dass eine Hauptaufgabe der Rechnungslegung Spenden sammelnder Organisationen nicht in der Gewinnermittlung besteht, sondern darin, der interessierten Öffentlichkeit und insbesondere den Spendern ein zutreffendes Bild über das Spendenaufkommen und dessen satzungsmäßiger und zweckentsprechender Verwendung zu vermitteln. Dies kann in Abhängigkeit von der Rechtsform der Organisation durch einen am Handelsrecht orientierten Jahresabschluss oder durch eine EinnahmenAusgaben-Rechnung mit Vermögensrechnung erfolgen.

Die neue Stellungnahme ersetzt die bisherige Verlautbarung (HFA 4/1995), geht jedoch inhaltlich teilweise vollkommen neue und von der derzeitigen Praxis Spenden sammelnder Organisationen abweichende Wege, so beispielsweise durch die Empfehlung zur Gliederung der Gewinn- und Verlustrechnung $(\mathrm{GuV})$ nach dem Umsatzkostenverfahren (statt des bislang gebräuchlichen Gesamtkostenverfah- rens) oder den Zeitpunkt der Ertragsrealisierung der Spendenzuflüsse. Diese sollen künftig nicht mehr im Zeitpunkt des Zuflusses, sondern erst im Zeitpunkt der zweckentsprechenden Verwendung als Ertrag in der Gewinn- und Verlustrechnung ausgewiesen werden. Bei Zufluss sollen sie zunächst ohne Berührung der Gewinn- und Verlustrechnung in einem gesonderten Passivposten »Noch nicht verbrauchte Spendenmittel « erfasst werden. Es bleibt abzuwarten, ob die Praxis diesen Empfehlungen folgen wird.

Insbesondere vor dem Hintergrund der neuen Wege und des daraus für die betroffenen Organisationen resultierenden Konfliktpotentials (z. B. bei gleichzeitig bestehender Pflicht zur Anwendung der Krankenhaus-Buchführungs-Verordnung KHBV oder Pflege-Buchführungsverordnung PBV) sowie des Aufwandes für eine Umstellung des Rechnungswesens auf das Umsatzkostenverfahren (insbesondere kleinere Organisationen dürften hier überfordert sein) stellt sich die Frage, welche Organisationen letztlich als Adressaten von der neuen Stellungnahme betroffen sind.

Zum Adressatenkreis wird in der Stellungnahme ausgeführt: »Eine juristische Person, die in ihrer Gesamtheit nicht den Charakter einer Spenden 
sammelnden Organisation aufweist, ist nicht verpflichtet, diese IDW-Stellungnahme zur Rechnungslegung zu befolgen. «Weiter heißt es: » Juristische Personen, deren Spendenerträge im Verhältnis zu ihren übrigen Erträgen unwesentlich sind und die daher nicht den Charakter einer Spenden sammelnden Organisation i. S. dieser IDW-Stellungnahme zur Rechnungslegung haben, können rechtlich unselbstständige Untereinheiten umfassen, die für sich genommen in einem wesentlichen Umfang Spenden sammeln und verwenden. In solchen Fällen empfiehlt es sich, dass die Untereinheit freiwillig gesondert Rechnung legt und dabei die in dieser IDW-Stellungnahme zur Rechnungslegung dargestellten Grundsätze anwendet." Problematisch ist in diesem Zusammenhang, dass eindeutige Definitionen der Begrifflichkeiten »in ihrer Gesamtheit « oder " unwesentlich « fehlen und damit eine eindeutige Abgrenzung erschwert wird. Eindeutige absolute oder relative Grenzwerte bestehen nicht.

Die Stellungnahme stellt aber mit der Formulierung »in ihrer Gesamtheit « sehr deutlich darauf ab, dass der Spenden sammelnde Bereich prägend für die Organisation sein muss. Daraus kann im Umkehrschluss abgeleitet werden, dass Organisationen, die ihre Arbeit überwiegend (d. h. zu mehr als $50 \%$ ) aus Leistungsentgelten finanzieren, in ihrer Gesamtheit nicht den Charakter einer Spenden sammelnden Organisation aufweisen und somit nicht Adressaten der Stellungnahme sind. Der Spenden sammelnde Charakter ist für sie in ihrer Gesamtheit nicht prägend, auch wenn Spenden für die Aufgabenerfüllung wichtig sind oder einzelne satzungsgemäße Aufgaben nur durch den Einsatz von Spenden erfüllt werden können. Damit gehören soziale Einrichtungen wie Krankenhäuser, Pflegeeinrichtungen, Einrichtungen der Behinderten- und Jugendhilfe, aber auch die Gliederungen der Wohlfahrtsverbände (z. B. Kreis- oder Stadtverbände), die ihre Aktivitäten regelmäßig überwiegend durch Leistungsentgelte oder zweckgebundene Zuschüsse finanzieren, nicht zum Adressatenkreis der Stellungnahme.

Etwas anderes gilt allenfalls dann, wenn die Organisation eine rechtlich unselbständige Untereinheit umfasst, die sich maßgeblich aus Spenden finanziert und im Außenverhältnis eigenständig wahrgenommen wird (z. B. Brot für die Welt, caritas international, DRK-Katastrophenhilfe, die rechtlich unselbständige Tätigkeitsbereiche ihrer Trägerorganisationen sind). Für diese Fälle empfiehlt die Stellungnahme, dass für diese Untereinheit freiwillig gesondert unter Beachtung der IDW-Stellungnahme Rechnung gelegt wird. Dies kann auch in Form einer Nebenrechnung erfolgen.

\section{Schlussfolgerungen}

Für Organisationen, deren Spendenaufkommen von geringerer Bedeutung ist, die in ihrer Gesamtheit nicht den Charakter einer Spenden sammelnden aufweisen und die demnach nicht Adressaten der Stellungnahme sind, ergibt sich daraus: Die Zuflüsse an Spenden und ähnlichen Erträgen (z. B. Förderbeiträge) können je nach Umfang unter Beibehaltung des Gesamtkostenverfahrens als Ertrag in einem gesonderten Posten nach den Umsatzerlösen oder unter den sonstigen betrieblichen Erträgen ausgewiesen werden. Im letzteren Fall empfiehlt sich ein DavonVermerk oder eine gesonderte Angabe der Spendenerträge im Anhang. Ergänzend können die zum Bilanzstichtag noch nicht zweckentsprechend verwendeten Spenden in der Bilanz nach dem Eigenkapital in einem gesonderten Posten »Noch zweckentsprechend zu verwendende Spenden « ausgewiesen werden.

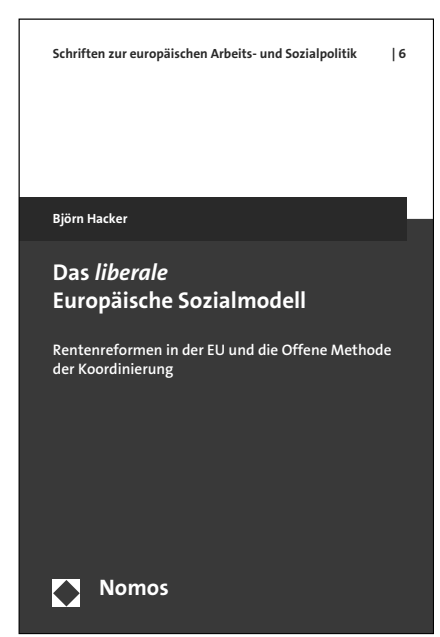

\section{Das liberale Europäische Sozialmodell}

Rentenreformen in der EU und die Offene Methode der Koordinierung Von Dr. Björn Hacker 2010, 428 S., brosch., 79,- $€$ ISBN 978-3-8329-5904-3 (Schriften zur europäischen Arbeits- und Sozialpolitik, Bd. 6)

Die Wohlfahrtsstaaten der EU stehen unter dem Anpassungsdruck der weit fortgeschrittenen Wirtschaftsintegration, während die soziale Dimension Europas gering ausgebaut bleibt. Diese Studie evaluiert erstmals die Relevanz europäischer Politikkoordinierung für nationale Reformvorhaben im Rentensektor und verortet ihre Rolle in einem Europäischen Sozialmodell.

\section{Nomos}

